\title{
Expression of Transcripts for the Vesicular Glutamate Transporters in the Human Medial Temporal Lobe
}

\author{
ROBERT E. MCCULLUMSMITH AND JAMES H. MEADOR-WOODRUFF \\ Department of Psychiatry and Mental Health Research Institute, \\ University of Michigan Medical School, Ann Arbor, Michigan 48109-0720, USA
}

\begin{abstract}
KEYWORDS: vesicular glutamate transporters; medial temporal lobe; in situ hybridization
\end{abstract}

Originally characterized as plasma membrane inorganic phosphate transporters, the vesicular glutamate transporters (vGluts) facilitate proton-dependent uptake of glutamate into presynaptic vesicles. ${ }^{1-3}$ In contrast to the plasma membrane-localized excitatory amino acid transporters, vGlut1 (BNPi) and vGlut2 (DNPi) do not transport aspartate or rely on a $\mathrm{Na}^{+}$gradient for glutamate transport. ${ }^{1}$ vGlut2, but not vGlut1, transcript expression is widespread in subcortical structures, while both are expressed in the cortex and striatum. ${ }^{2-4}$ Interestingly, a third vesicular transporter, vGlut3, was recently characterized and reportedly expressed in serotonergic and cholinergic neurons. ${ }^{5-7}$ Differential patterns of distribution of vGlut mRNAs likely define subtypes of glutamatergic neurons, potentially yielding novel information regarding glutamatergic circuitry. For this study, we utilized in situ hybridization to characterize transcript expression of vGlut 1,2, and 3 in medial temporal lobe structures to determine if subpopulations of glutamatergic neurons exist in this brain region.

Human brain sections, provided by the Stanley Foundation Neuropathology Consortium, were obtained at autopsy and $14 \mu \mathrm{m}$ sections were prepared as previously described. ${ }^{8}$ To generate subclones we amplified unique regions of vGlut1 (NCBI Genebank ascension number AB032436, nucleotide coding region 953-1481), vGlut2 (AB032435, 1337-1843), and vGlut3 (NM_139319, 826-1673) from a human cDNA brain library using PCR. Amplified cDNA segments were extracted, subcloned and confirmed by nucleotide sequencing. Riboprobes were synthesized from linearized plasmid DNA and in situ hybridization was performed as previously described.$^{9,10}$ Radiolabeled sections were dipped in NTB-2 emulsion, incubated for $2-$ 12 weeks, developed, and counterstained as previously described. ${ }^{11}$

Address for correspondence: Robert E. McCullumsmith, M.D., Ph.D., Mental Health Research Institute and Department of Psychiatry, University of Michigan, 205 Zina Pitcher Place, Ann Arbor, MI 48109-0720. Voice: 734-936-2061; fax: 734-647-4130.

smithrob@umich.edu

Ann. N.Y. Acad. Sci. 1003: 438-442 (2003). @ 2003 New York Academy of Sciences. doi: 10.1196/annals.1300.046 


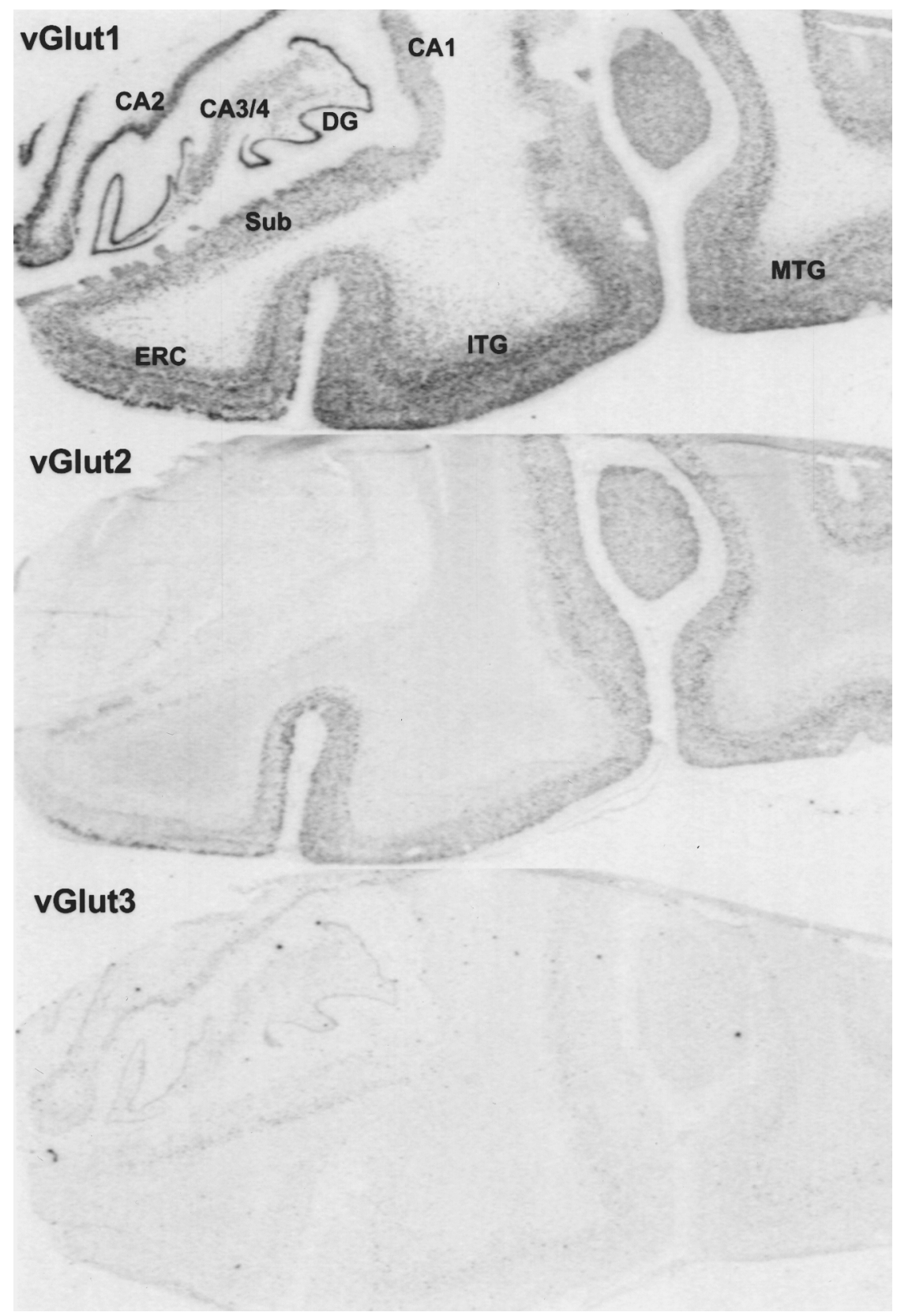

FIGURE 1. In situ hybridization with $\left[{ }^{35} \mathrm{~S}\right]$-riboprobes demonstrating vGlut 1,2 , and 3 transcript expression in the human medial temporal lobe. Abbreviations: vesicular glutamate transporter (vGlut), dentate gyrus (DG), subiculum (Sub), entorhinal cortex (ERC), inferior temporal gyrus (ITG), middle temporal gyrus (MTG). 
vGlut1 transcript expression was apparent after a 3-day exposure of [ $\left.{ }^{35} \mathrm{~S}\right]$-radiolabeled sections exposed to film, versus a 6-week exposure for vGlut 2 and vGlut3. We detected vGlut1 and vGlut3 transcript expression in the hippocampal subfields CA1, CA2, CA3, and CA4, the dentate gyrus (DG), the subiculum (Sub), the entorhinal cortex (ERC), the inferior temporal gyrus (ITG), and the middle temporal gyrus (MTG) (FIG. 1). vGlut2 transcripts were detected in DG, Sub, ERC, ITG,
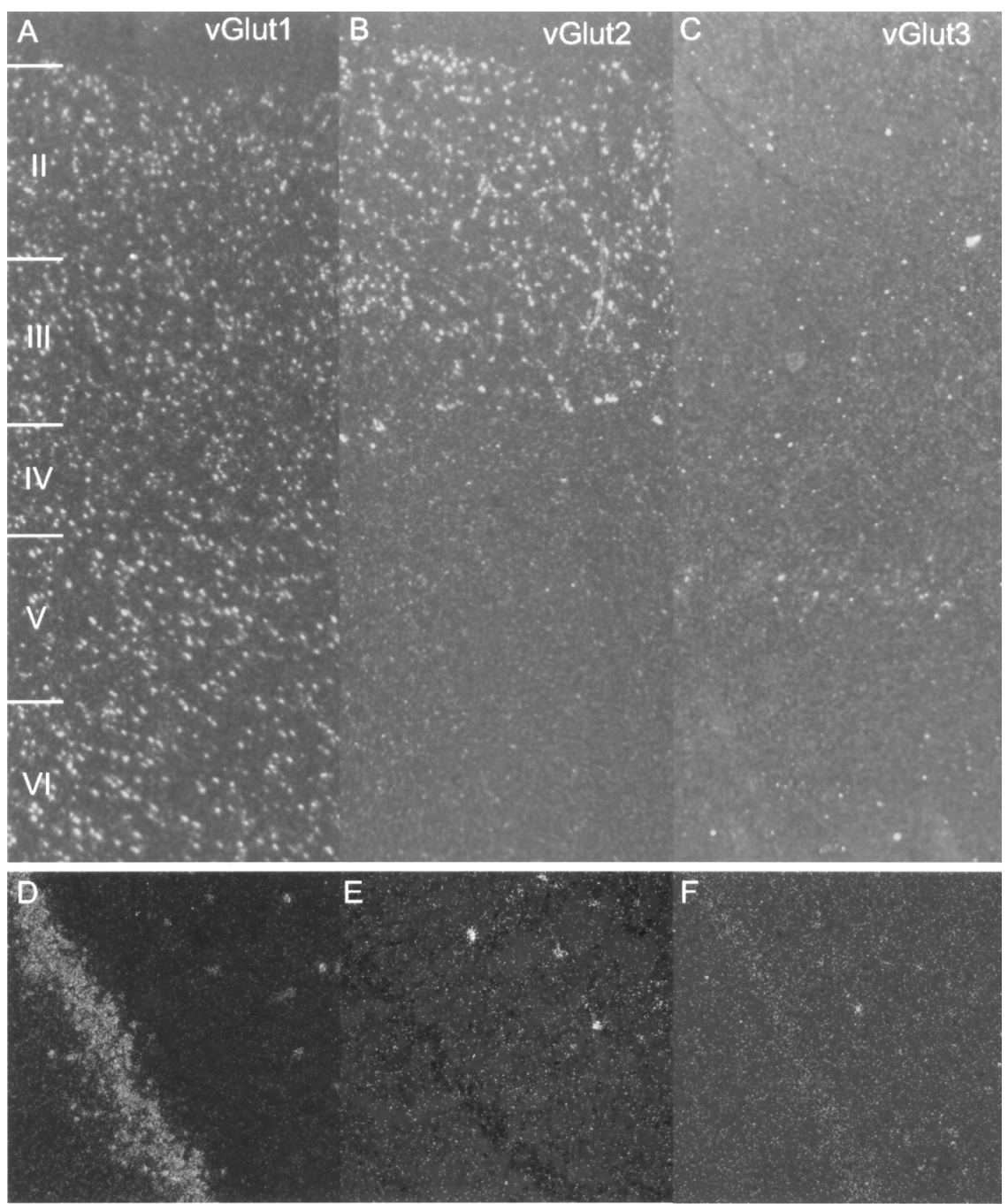

FIGURE 2. Darkfield microscopy of $\left[{ }^{35} \mathrm{~S}\right]$-radiolabeled vGlut1 (A, D), vGlut2 (B, E), and vGlut3 $(\mathbf{C}, \mathbf{F})$ transcripts in emulsion dipped slides. Cortical layers of the inferior temporal gyrus $(\mathbf{A}-\mathbf{C})$ and identification of the dentate gyrus $(\mathbf{D}-\mathbf{F})$ were confirmed by Nissl counterstaining. 
MTG and also in scattered neurons of CA4 (FIGS. 1 and 2). Cortical vGlut1 transcripts were differentially expressed in layers II-VI of the ERC, ITG, and MTG, while vGlut 2 expression was primarily restricted to the superficial pyramidal cell layers (II and III) in these same cortical regions (FIGS. 1 and 2). Cortical vGlut3 expression was primarily limited to layer V in the ITG and MTG, and layer II in the ERC (FIGS. 1 and 2). The level of expression of vGlut 1, 2, and 3 mRNAs in the DG exemplifies the differential distribution of transcripts for these genes in the medial temporal lobe (Fig. 2, D-F).

We have detected unique patterns of expression of vGlut 1,2, and 3 transcripts in the medial temporal lobe, consistent with previous reports demonstrating expression of vGlut 1 in the cortex and hippocampus, and vGlut 2 in the cortex. ${ }^{2-4}$ Interestingly, vGlut2 transcript expression was restricted to superficial pyramidal cell layers in the cortex, suggesting an association with glutamatergic neurons in layers II and III that primarily form cortico-cortical connections. In contrast, vGlut1 was expressed in both superficial and deeper cortical layers. Evaluation of emulsion-dipped sections revealed that expression of vGlut 3 mRNA in the cortex is primarily in large pyramidal neurons and is restricted in large part to layer V of the ITG and MTG, suggesting an association with glutamatergic neurons that project to subcortical structures. These findings support the hypothesis that the vGluts probably define subpopulations of glutamatergic neurons and are likely associated with specific efferent pathways and circuits.

\section{ACKNOWLEDGMENTS}

This work was supported by a Pfizer Postdoctoral Fellowship (R.E.M.) and MH53327 (J.M.W.).

\section{REFERENCES}

1. Bellocchio, E.E., et al. 2000. Uptake of glutamate into synaptic vesicles by an inorganic phosphate transporter. Science 289: 957-960.

2. Fremeau, R.T., JR., et al. 2001. The expression of vesicular glutamate transporters defines two classes of excitatory synapse. Neuron 31: 247-260.

3. Fujiyama, F., T. Furuta \& T. KANEKO. 2001. Immunocytochemical localization of candidates for vesicular glutamate transporters in the rat cerebral cortex. J. Comp. Neurol. 435: 379-387.

4. Bellocchio, E.E., et al. 1998. The localization of the brain-specific inorganic phosphate transporter suggests a specific presynaptic role in glutamatergic transmission. J. Neurosci. 18: 8648-8659.

5. FremeAu, R.T., JR., et al. 2002. The identification of vesicular glutamate transporter 3 suggests novel modes of signaling by glutamate. Proc. Natl. Acad. Sci. USA 99: 14488-14493.

6. TAKAMORI, S. et al. 2002. Molecular cloning and functional characterization of human vesicular glutamate transporter 3. EMBO Rep. 3: 798-803.

7. Gras, C. et al. 2002. A third vesicular glutamate transporter expressed by cholinergic and serotoninergic neurons. J. Neurosci. 22: 5442-5451.

8. TORREY, E.F. et al. 2000. The Stanley Foundation brain collection and neuropathology consortium. Schizophr. Res. 44: 151-155.

9. Sмith, R.E. et al. 2001. Vesicular glutamate transporter transcript expression in the thalamus in schizophrenia. Neuroreport 12: 2885-2887. 
10. McCullumsmith, R.E. \& J.H. Meador-Woodruff. 2002. Striatal excitatory amino acid transporter transcript expression in schizophrenia, bipolar disorder, and major depressive disorder. Neuropsychopharmacology 26: 368-375.

11. MEAdor-Woodruff, J.H. et al. 1989. Distribution of D2 dopamine receptor mRNA in rat brain. Proc. Natl Acad Sci. USA 86: 7625-7628. 